\title{
Disordered double Weyl node: Comparison of transport and density of states calculations
}

\author{
Björn Sbierski, ${ }^{1, *}$ Maximilian Trescher, ${ }^{1}$ Emil J. Bergholtz, ${ }^{1,2}$ and Piet W. Brouwer ${ }^{1}$ \\ ${ }^{1}$ Dahlem Center for Complex Quantum Systems and Institut für Theoretische Physik, Freie Universität Berlin, D-14195 Berlin, Germany \\ ${ }^{2}$ Department of Physics, Stockholm University, SE-106 91 Stockholm, Sweden
}

(Received 23 December 2015; revised manuscript received 9 February 2017; published 1 March 2017)

\begin{abstract}
Double Weyl nodes are topologically protected band crossing points which carry chiral charge \pm 2 . They are stabilized by $C_{4}$ point-group symmetry and are predicted to occur in $\mathrm{SrSi}_{2}$ or $\mathrm{HgCr}_{2} \mathrm{Se}_{4}$. We study their stability and physical properties in the presence of a disorder potential. We investigate the density of states and the quantum transport properties at the nodal point. We find that, in contrast to their counterparts with unit chiral charge, double Weyl nodes are unstable to any finite amount of disorder and give rise to a diffusive phase, in agreement with the predictions of Goswami and Nevidomskyy [Phys. Rev. B 92, 214504 (2015)] and Bera, Sau, and Roy [Phys. Rev. B 93, 201302 (2016)]. However, for finite system sizes a crossover between pseudodiffusive and diffusive quantum transport can be observed.
\end{abstract}

DOI: 10.1103/PhysRevB.95.115104

\section{INTRODUCTION}

Topological metals and semimetals are among the driving themes in contemporary condensed matter physics. Their most prominent three-dimensional realizations are Weyl (semi-)metals, which have recently been experimentally confirmed in a number of material systems [1-6]. Pioneering experimental studies used spectroscopic measurements to study surface Fermi arcs and characteristic Weyl node bulk dispersions. Recently, also (magneto-)transport properties have received growing interest in experiments [7-9].

While the sample quality matures continuously, more controlled engineering of the chemical potential becomes possible[10,11]. Weyl nodes with chemical potential $\mu$ sufficiently close to the nodal point $(\mu=0)$ are predicted to show unusual transport characteristics for sample length $L \lesssim \hbar v / \mu$, with $v$ the Fermi velocity $[12,13]$. Without disorder, the conductance scales with the system size as $G \propto W^{2} / L^{2}$, where $W$ is the sample width. The inclusion of weak disorder is irrelevant in the renormalization-group sense [14-19] and consequently does not change the size dependence of the conductance. This so-called "pseudoballistic" regime is further characterized by an unusual Fano factor (the ratio of shot-noise power to average current), $F \approx 0.57[12,13]$. Only if disorder increases above a critical strength do the conductivity and density of states at the nodal point attain a nonzero value and does transport become diffusive, $G \propto W^{2} / L$ and $F=1 / 3$.

The simple Weyl node (SWN) band structure discussed above carries a topological charge of \pm 1 . Beyond the SWN, the existence of topological band touching points with a higher topological charge is tied to the presence of point-group symmetries [20]. In this paper, we consider double Weyl nodes (DWNs) with a chiral charge of magnitude 2 , stabilized by $C_{4}$ rotation symmetry. The Hamiltonian reads

$$
H=\hbar v\left[\sigma_{x} \eta_{x}\left(k_{x}^{2}-k_{y}^{2}\right) / 2+\sigma_{y} \eta_{y} k_{x} k_{y}+k_{z} \sigma_{z}\right],
$$

with $\eta_{x, y}$ internal length scales. The fourfold rotational symmetry around the $z$ axis is realized as $H\left(k_{x}, k_{y}, k_{z}\right)=$ $\sigma_{z} H\left(k_{y},-k_{x}, k_{z}\right) \sigma_{z}$. Time-reversal symmetry is present,

\footnotetext{
*b.sbierski@fu-berlin.de
}

$\sigma_{x} H^{*}(-\mathbf{k}) \sigma_{x}=H(\mathbf{k})$, with the time reversal operator $T=$ $\sigma_{x} K$ squaring to +1 . To simplify the subsequent analysis, we specialize to the case $\eta_{x}=\eta_{y} \equiv \eta$, where the discrete rotation symmetry is extended to a continuous rotation symmetry $C_{\infty}$, in cylindrical coordinates $H\left(k_{\perp}, \phi, k_{z}\right)=$ $e^{-i \theta \sigma_{z}} H\left(k_{\perp}, \phi-\theta, k_{z}\right) e^{i \theta \sigma_{z}}$. The corresponding energy dispersion $\varepsilon_{ \pm}^{2} /(\hbar v)^{2}=\left(k_{\perp}^{2} \eta / 2\right)^{2}+k_{z}^{2}$ is quadratic in the momentum $\mathbf{k}_{\perp}=\left(k_{x}, k_{y}\right)$ transverse to the rotation axis and linear in $k_{z}$ [see Fig. 1(a)]. A photonic crystal realization of DWNs is reported in Ref. [21] and fermionic candidate materials have been identified from first-principle calculations, such as $\mathrm{HgCr}_{2} \mathrm{Se}_{4}$ [22] and $\mathrm{SrSi}_{2}$ [23]. The latter material might be experimentally more feasible since no magnetic ordering is required. An interesting proposal of detecting the monopole charge in electronic Weyl materials using transport measurements has recently been formulated in Ref. [24].

In view of the requirement of point-group symmetries, the stability of a DWN to disorder, which typically breaks such symmetry [see Fig. 1(b)], is a relevant question. Several groups have addressed this question theoretically, with partially diverging results. Using a simplified version of the self-consistent Born approximation (SCBA), Goswami and Nevidomskyy [25] argued that the DWN is unstable to disorder and that the inclusion of even a small amount of disorder drives the system to a diffusive phase with zero-energy scattering rate $\hbar / \tau \sim e^{-A / K}$, where $K$ is a dimensionless measure of the disorder strength and $A$ a material-dependent parameter. The same conclusion was drawn by Bera, Sau, and Roy [26], based both on a renormalization-group analysis [which found disorder a marginally relevant perturbation to Eq. (1)] and on a numerical calculation of the density of states at zero energy, which was claimed to be compatible with the exponential form proposed above.

Recently, Shapourian and Hughes [27] revisited the same problem, conducting a finite-size scaling analysis of the decay length in the $z$ direction using a transfer-matrix method. Their data indicate the presence of a critical point at a finite disorder strength (but below the Anderson transition), leading them to conclude the stability of the DWN phase against weak disorder. A possible scenario for such an observation would be the splitting of the DWN into two equally charged SWNs under the influence of disorder, where the latter individually would 
(a)

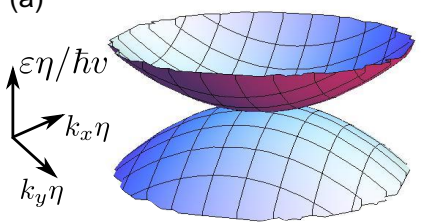

(b)

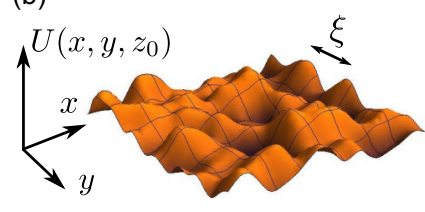

FIG. 1. (a) Dispersion for the DWN Hamiltonian $H$ with $\eta_{x, y}=\eta$ in Eq. (1) at $k_{z}=0$. (b) The potential disorder profile is characterized by its Gaussian correlations' decaying on a length scale $\xi$; here a slice at $z=z_{0}$ is shown.

indeed feature a critical point. This interesting scenario and the apparent contradiction between results in the literature motivated us to revisit the problem of a disordered DWN.

We first investigate the density of states using the kernel polynomial method (KPM) and the self-consistent Born approximation (Sec. II). We discuss the shortcomings of both methods and move on to a scattering-matrix-based transport calculation, much better suited to study the physics right at the nodal point (Sec. III). These combined numerical efforts allow us to put forward the following interpretation: In the presence of any finite amount of disorder, the clean DWN fixed point is unstable and gives rise to a diffusive phase. We find no evidence in support of a critical point at finite disorder strength or, accordingly, of the DWN splitting scenario. However, due to the exponentially low scattering rate, a crossover behavior can be observed in the quantum transport properties of weakly disordered mesoscopic samples.

\section{DENSITY OF STATES}

\section{A. Kernel polynomial method}

We start by calculating the density of states in a disordered DWN which we regularize on a cubic lattice,

$$
\begin{aligned}
H_{\mathrm{L}}(\mathbf{k})= & \varepsilon_{0} \frac{\eta}{a}\left[\sigma_{x}\left(\cos a k_{x}-\cos a k_{y}\right)+\sigma_{y} \sin a k_{x} \sin a k_{y}\right] \\
& -\varepsilon_{0} \sigma_{z} \cos a k_{z},
\end{aligned}
$$

where $\varepsilon_{0}=\hbar v / a$ and $a$ is the lattice constant. The effective low-energy approximation of $H_{\mathrm{L}}$ around $\varepsilon=0$ consists of four DWNs centered at $k_{z}= \pm \frac{\pi}{2 a}$ and $\left(k_{x}, k_{y}\right)=(0,0)$ or $\left(\frac{\pi}{a}, \frac{\pi}{a}\right)$, with minimal distance $\Delta k=\pi / a$. We include a Gaussian disorder potential $U(\mathbf{r})$ characterized by zero mean and realspace correlations given by

$$
\left\langle U(\mathbf{r}) U\left(\mathbf{r}^{\prime}\right)\right\rangle_{\mathrm{dis}}=\frac{K(\hbar v)^{2}}{\sqrt{2 \pi}^{3} \xi^{2}} e^{-\left|\mathbf{r}-\mathbf{r}^{\prime}\right|^{2} / 2 \xi^{2}},
$$

where $\xi$ is the correlation length and $K$ the dimensionless disorder strength. In the following, we use $\xi=\eta / 2$, but different choices do not qualitatively change our conclusions. To smoothly represent $U(\mathbf{r})$ on the lattice scale, we take $\xi=2.9 a$, which suppresses the internode scattering rate by a factor $e^{-(\Delta k)^{2} \xi^{2} / 2}<10^{-18}$ compared to the intranode rate, so single-node physics (i.e., $H+U$ ) is realized to a very good approximation.

We numerically calculate the density of states of $H_{\mathrm{L}}+U(\mathbf{r})$ using the KPM (see Ref. [28] for a description of the method). The resulting density of states normalized to a single DWN is
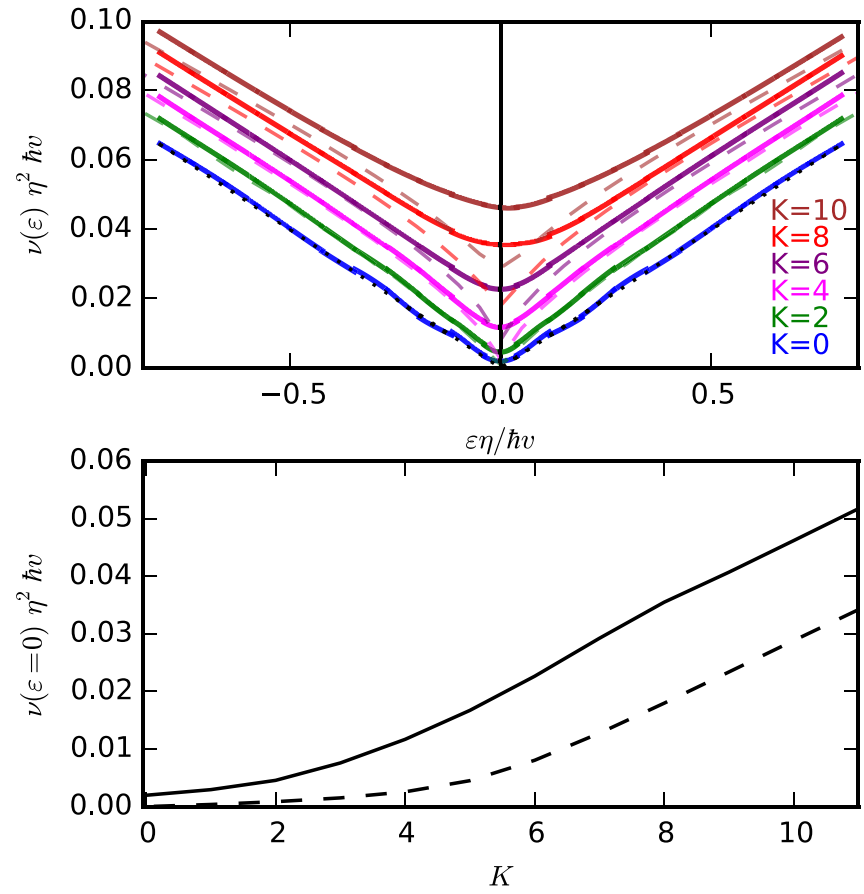

FIG. 2. Density of states $v$ as a function of the energy $\varepsilon$ (top) and at the nodal point $\varepsilon=0$ (bottom) normalized to a single DWN computed from the kernel polynomial method (KPM) applied to the lattice Hamiltonian $H_{\mathrm{L}}$ (solid lines). The results of the self-consistent Born approximation based on $H$ (dashed lines) are in good agreement with the KPM data except in the vicinity of the nodal point and for high disorder strengths $K>6$. We take the disorder correlation length $\xi=\eta / 2$. The system size of the tight-binding model underlying the KPM calculation is $L_{x, y}=100 a, L_{z}=260 a$, and we apply periodic boundary conditions. The expansion order in Chebyshev polynomials $N$ is taken between 1000 and 6000 depending on the energy $\varepsilon$, so that $v$ is minimized but oscillations due to the underlying discrete eigenenergies of the finite system are sufficiently smoothed out. An average over 10 disorder realizations is taken and 20 random vectors are used to calculate the trace in the KPM.

shown as solid lines in Fig. 2(a). Further simulation parameters are given in the figure caption. The analytical result for an infinite clean system,

$$
\nu_{0}(\varepsilon)=\frac{\varepsilon}{4 \pi(\hbar v)^{2} \eta},
$$

is shown as the dotted line in Fig. 2 and compares well with the $K=0 \mathrm{KPM}$ results except at $\varepsilon=0$. At the nodal point, the KPM method has intrinsic difficulties to simulate the vanishing (or very low) density of states, which is due to the finite expansion order of $v(\varepsilon)$ in Chebyshev polynomials and the discrete nature of eigenstates in a finite tight-binding model. In Fig. 2(b), we plot $v(\varepsilon=0)$ vs $K$. Our findings are in qualitative agreement with similar numerical results in Ref. [26]: The presence of disorder scattering fills the dip in the density of states for any finite disorder strength.

\section{B. Self-consistent Born approximation}

A frequently employed analytical approach to disordered electronic systems is the self-consistent Born approximation. 
Although a simplified SCBA calculation was performed in Ref. [25], in the following we compute the SCBA self-energy for $H+U$ and the associated density of states without any further approximations. The results are shown as dashed lines in Fig. 2; comparison to the KPM results confirms that the SCBA is accurate only at high energies $\varepsilon$ and weak disorder values when $\varepsilon \tau \gg \hbar$, with $\tau$ being the quasiparticle scattering time.

We start from Hamiltonian $H$ with $\eta_{x}=\eta_{y} \equiv \eta$ and seek to describe the disorder-averaged retarded Green function $\left\langle G^{R}\right\rangle_{\text {dis }}=1 /\left(\varepsilon-H-\Sigma^{R}\right)$ in terms of a translationally invariant self-energy term $\Sigma^{R}$ that fulfills the SCBA equation,

$$
\Sigma^{R}(\mathbf{k})=\int \frac{d \mathbf{k}^{\prime}}{(2 \pi)^{3}}\left\langle G^{R}\left(\mathbf{k}^{\prime}\right)\right\rangle_{\mathrm{dis}}\left|U\left(\mathbf{k}^{\prime}-\mathbf{k}\right)\right|^{2},
$$

where $\left|U\left(\mathbf{k}^{\prime}-\mathbf{k}\right)\right|^{2}$ is the Fourier transform of the disorder correlator in Eq. (3). Since a disorder average restores the $C_{\infty}$ symmetry of the system around the $k_{z}$ axis, the projection of $\Sigma^{R}\left(k, \phi, k_{z}\right)$ to the $\sigma_{x}-\sigma_{y}$ plane in Pauli-matrix space should point in the $\phi$ direction, the angle between this plane and the $\sigma_{z}$ projection of $\Sigma^{R}\left(k, \phi, k_{z}\right)$ is not dictated by symmetry and can be different from the angle in $H\left(k, \phi, k_{z}\right)$. With these considerations, a natural ansatz for the self-energy is

$$
\begin{aligned}
\Sigma^{R}\left(k, \phi, k_{z}\right) / \hbar v= & m\left(k, k_{z}\right)\left(\cos [2 \phi] \sigma_{x}+\sin [2 \phi] \sigma_{y}\right) \\
& +\sigma_{z} m_{z}\left(k, k_{z}\right)-i m_{0}\left(k, k_{z}\right),
\end{aligned}
$$

with $m, m_{z}$, and $m_{0}$ complex and $\operatorname{Re}\left[m_{0}\right]>0$. At $\varepsilon=0$, in order to avoid the unphysical spontaneous generation of a chemical potential from disorder with $\langle U(\mathbf{r}))\rangle_{\text {dis }}=0, m_{0}$ has to be chosen purely real, which requires also $m$ and $m_{z}$ to be real quantities. The resulting self-consistency equations for $m, m_{z}$, and $m_{0}$ are given in the Appendix and can be solved numerically by iteration. The density of states follows from

$$
\nu(\varepsilon)=-\frac{1}{\pi} \operatorname{Im} \int \frac{d \mathbf{k}}{(2 \pi)^{3}} \operatorname{Tr}\left\langle G^{R}(\mathbf{k})\right\rangle_{\mathrm{dis}} .
$$

Results of this calculation are shown as dashed lines in Fig. 2 for various representative disorder strengths $K$.

\section{Discussion}

The SCBA calculation in Sec. II can be simplified by taking the disorder correlation length to 0 and choosing a finite (half-)band width, $\Lambda \gg \hbar v / \eta$. Then we can define $K$ such that $\left|U\left(\mathbf{k}^{\prime}-\mathbf{k}\right)\right|^{2}=K \Lambda^{2} \eta^{3}$ and insert this into Eq. (5) at $\varepsilon=0$, where $\Sigma^{R} \equiv-i \Gamma$ becomes independent of $\mathbf{k}$. Transforming to an energy integral and using the density of states, (4), along with the assumption $\Gamma \ll \Lambda$, one finds

$$
\Gamma=\Lambda e^{-A / K}
$$

with $A=2 \pi(\hbar v / \Lambda \eta)^{2}$. This was first observed by Goswami and Nevidomskyy in Ref. [25] and states that any finite disorder strength gives rise to a finite lifetime $1 / \Gamma$ of quasiparticles and a finite density of states $\nu(\varepsilon=0) \propto \Gamma$ at the nodal point. Our SCBA analysis, which takes into account a more realistic disorder model and infinite bandwidth, confirms the simplified result in Eq. (8) qualitatively (see dashed line in Fig. 2, bottom).

However, it is well known that the SCBA is not reliable around gapless points, where the smallness of the parameter
$k_{F} l$ spoils the suppression of crossed diagram contributions to the self-energy (see, e.g., Ref. [13] for a discussion in the context of simple Weyl nodes). Indeed, comparing the nonperturbative KPM results for $v(\varepsilon)$ to the SCBA in Fig. 2, good agreement is achieved away from the nodal point only. At the nodal point, it is difficult to judge the qualitative validity of Eq. (8) based on the KPM results. The reason is that, for the latter method, finite size and smoothing effects tend to overestimate $v(\varepsilon=0)$. [For example, the KPM method returns a finite value of $v(\varepsilon=0)$ even for $K=0$ (see Fig. 2, bottom)]. In summary, neither numerical nor analytical calculations of the density of states as presented above are conclusive in gauging the qualitative validity of Eq. (8) against the alternative scenario of a finite critical disorder strength below which the bulk density of states vanishes. In this situation, we switch to a quantum transport framework which is ideally suited to study the disordered DWN at the nodal point.

\section{QUANTUM TRANSPORT}

\section{A. Clean case}

We start this section by calculating the conductance and shot-noise power of a clean mesoscopic DWN sample of length $L$ and width $W$ coupled to ideal leads, building on earlier work by Tworzydlo et al. on two-dimensional Dirac nodes [29]. We choose the transport direction as the $z$ direction and place the chemical potential at the nodal point. We model the leads as highly doped DWNs, $H_{\text {lead }}=H+V$ with $V \rightarrow \infty$. By matching wave functions at the sample-lead interfaces we calculate the transmission amplitudes $t_{0}\left(\mathbf{k}_{\perp}\right)$ and $t_{0}^{\prime}\left(\mathbf{k}_{\perp}\right)$ and reflection amplitudes $r_{0}\left(\mathbf{k}_{\perp}\right)$ and $r_{0}^{\prime}\left(\mathbf{k}_{\perp}\right)$, where the primed (unprimed) amplitudes refer to electrons incident from the positive (negative) $z$ direction,

$$
\begin{aligned}
& t_{0}=t_{0}^{\prime}=1 / \cosh \left(\eta L k_{\perp}^{2} / 2\right), \\
& r_{0}=-r_{0}^{\prime *}=i e^{-2 i \varphi} \tanh \left(\eta L k_{\perp}^{2} / 2\right),
\end{aligned}
$$

$\mathbf{k}_{\perp}=\left(k_{x}, k_{y}\right)$ the transverse component of the wave vector, and $\varphi=\arctan \left(k_{y} / k_{x}\right)$ is the azimuthal angle of incidence. The associated basis spinors for propagating states in the lead are $(0,1)^{\mathrm{T}}$ and $(1,0)^{\mathrm{T}}$ for left- and right-moving modes, respectively. From the transmission amplitude $t_{0}\left(\mathbf{k}_{\perp}\right)$ we compute the clean-limit conductance and Fano factor as $G_{0}=\frac{e^{2}}{h} \operatorname{tr}\left[t_{0}^{\dagger} t_{0}\right]$ and $F_{0}=\operatorname{tr}\left[t_{0} t_{0}^{\dagger}\left(1-t_{0} t_{0}^{\dagger}\right)\right] / \operatorname{tr}\left[t_{0} t_{0}^{\dagger}\right]$ [30]. Modes with $k_{\perp} \gg$ $(L \eta)^{-1 / 2} \equiv k_{\perp}^{\star}(L)$ are strongly suppressed in transmission and the spacing of the quantized transversal wave vectors in a finite sample is $\Delta k_{\perp}=2 \pi / W$. If $\Delta k_{\perp} \ll k_{\perp}^{*}$, we can compute the conductance and Fano factor analytically by replacing the sum over discrete modes $\mathbf{k}_{\perp}$ by an integral and find

$$
\begin{gathered}
G_{0}(W, L)=\frac{e^{2}}{h} \frac{1}{2 \pi \eta} \frac{W^{2}}{L}, \\
F_{0}(W, L)=1 / 3,
\end{gathered}
$$

which resembles transport in a diffusive conductor with conductivity $\sigma_{0}=e^{2} /(2 \pi h \eta)$. Thus, the clean DWN has pseudodiffusive transport characteristics-similar to Dirac electrons in two dimensions [29]. 


\section{B. Disordered case}

We extend the scattering matrix approach to include a Gaussian disorder potential $U(\mathbf{r})$ with correlations as in Eq. (3) and $\xi=\eta / 2$ as in the density of states calculation. We compute the transmission matrix of the disordered DWN $H+U(\mathbf{r})$ by concatenating the reflection and transmission amplitudes of a thin slice of DWN without disorder [see Eq. (9)] with the reflection and transmission matrices of a thin slice with disorder, which can be calculated using the first-order Born approximation, and repeating this procedure for many slices. We apply periodic or antiperiodic boundary conditions in the $x$ and $y$ directions, cutting off the number of transverse modes to keep the dimensions of the transmission and reflection matrices finite. We take the mode cutoff large enough and the slice length thin enough so that the results do not depend on either, and we take $\Delta k_{\perp} / k_{\perp}^{*}$ small enough that the results do not depend on the choice of the boundary conditions. A similar method has been previously applied to study disordered Dirac materials in two [31,32] and in three dimensions [13,33], and we refer the reader to these references for more details on the numerical method.

Figure 3 shows our results for the resistance $R=1 /\langle G\rangle_{\text {dis }}$ as a function of the sample length $L$, where $\langle\ldots\rangle_{\text {dis }}$ denotes an average over 60 disorder realizations as well as the two choices for the boundary conditions, to further suppress statistical uncertainty. Compared to the clean pseudoresistance $R_{0}=L /\left(\sigma_{0} W^{2}\right)$, the resistance of the disordered samples is slightly decreased, by up to about $10 \%$ (Fig. 3, top). The difference $\Delta R=R_{0}-R$ is shown in the bottom panel in

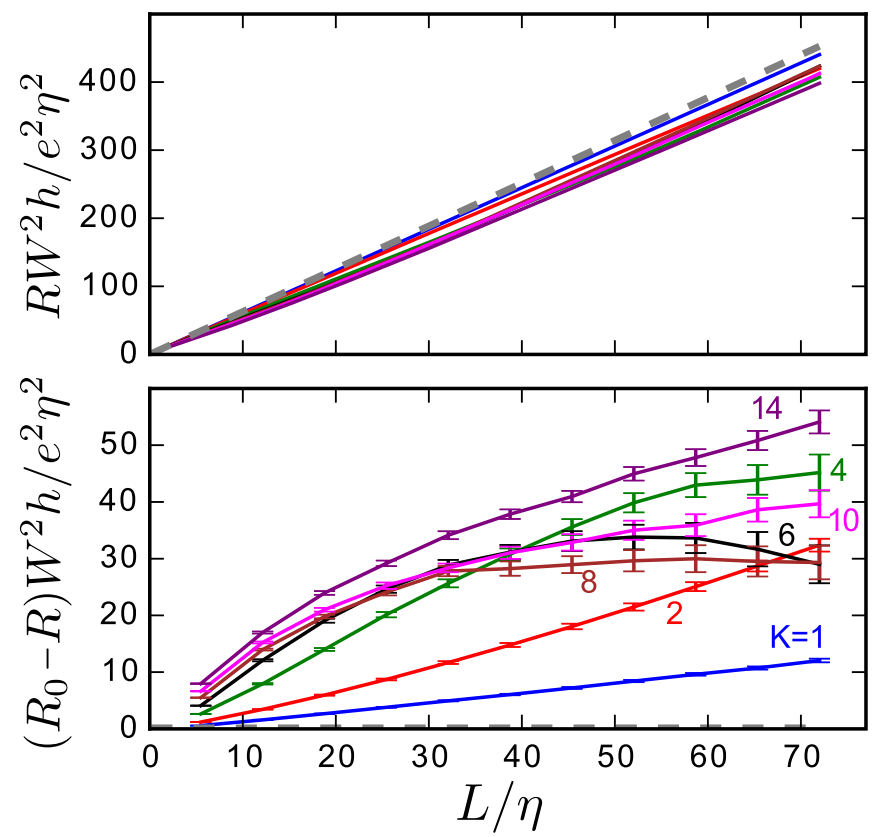

FIG. 3. Resistance for the disordered DWN for disorder strengths $K=1,2,4,6,8,10$, and 14 and disorder correlation length $\xi=$ $\eta / 2$. Results are averaged over periodic and antiperiodic transverse boundary conditions and over 60 disorder realizations per boundary condition. The dashed line indicates the clean-limit pseudodiffusive result of Eq. (10). We choose $W=72 \eta$ and keep transverse modes with $\left|k_{x, y}\right|<2 \pi M / W$ where $M=34$.
Fig. 3. For the lowest disorder strength considered, $K=1$, $\Delta R$ scales linearly with $L$ for the system lengths considered; for intermediate $K=2,4$, and $6, \Delta R$ is not a linear function of $L$ but instead has an " $S$ "-like dependence, which prevents any meaningful assignment of a (change in the) bulk resistivity. The resistance at the largest system size, $R\left(L_{\max }=72 \eta\right)$, shows nonmonotonous behavior with increasing disorder strength. For larger $K=8,10$, and 14 , the $\Delta R$ traces are purely convex and tend to be linear for large $L$. We have also investigated the Fano factor, which stays around $F=1 / 3$ (not shown) for all values of $K$.

\section{Discussion}

A finite lifetime $1 / \Gamma$ implies diffusive transport with resistance scaling $R \propto L$. While this is (approximately) observed in our transport simulations for $K>0$ (see Fig. 3, top), the difficulty lies in the discrimination between diffusive transport and transport associated to the clean fixed point $K=0$ : Being pseudodiffusive, the same resistance scaling holds, albeit for the very different reason of evanescent wave physics, and not due to scattering between transport channels as in diffusive transport. To discriminate between the pseudodiffusive and the diffusive regimes, in Fig. 4 (top) we show the probability $P_{\mathrm{t}}(L)$ that an electron is transmitted in the same transverse mode in which it entered-for which we take the mode with $\mathbf{k}_{\perp}=0$ - conditional on the probability that it is transmitted,

$$
P_{\mathrm{t}}(L)=\frac{|t(0,0)|^{2}}{\sum_{\mathbf{k}_{\perp}}\left|t\left(\mathbf{k}_{\perp}, 0\right)\right|^{2}},
$$
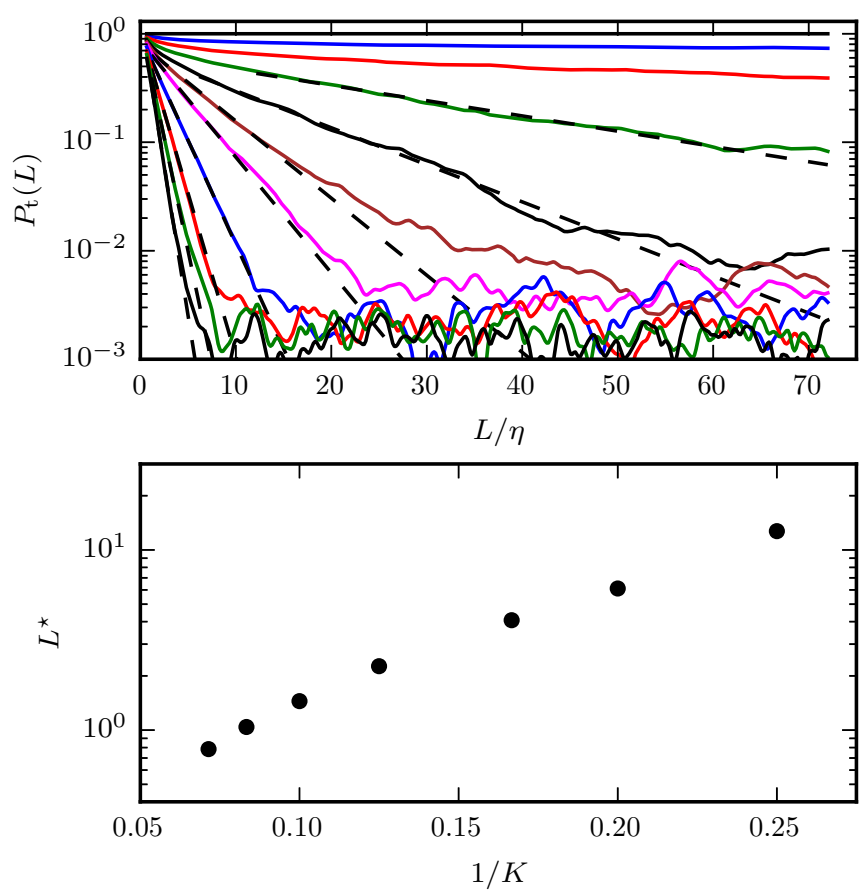

FIG. 4. Top: Conditional same-mode transmission probability $P_{\mathrm{t}}(L)$ for disorder strengths $K=0,1,2,3,4,5,6,8,10,12$, and 14 (top to bottom curves). Dashed lines denote fits to the form $\exp \left(-\left(L-L_{c}\right) / L^{\star}\right)$. Bottom: Mean free path $L^{\star}$, obtained from the fits to $P_{\mathrm{t}}(L)$, versus $1 / K$. 
where $t\left(\mathbf{k}_{\perp}^{\text {out }}, \mathbf{k}_{\perp}^{\text {in }}\right)$ is the transmission amplitude of the disordered system at length $L$, with $\mathbf{k}_{\perp}^{\text {out }}$ and $\mathbf{k}_{\perp}^{\text {in }}$ referring to the incoming and outgoing transverse modes.

The conditional probability $P_{\mathrm{t}}$ is an indicator of the transition between the pseudodiffusive and the regimes: At the pseudodiffusive fixed point $K=0$ one has $P_{\mathrm{t}}(L)=1$, as translational invariance ensures that $t\left(\mathbf{k}_{\perp}^{\text {out }}, \mathbf{k}_{\perp}^{\text {in }}\right)$ is diagonal in the transversal mode indices $\mathbf{k}_{\perp}^{\text {in }}$ and $\mathbf{k}_{\perp}^{\text {out }}$ [see (9)]. In contrast, diffusive transport is characterized by scattering between transverse modes. For sufficiently long diffusive samples with many transverse modes one therefore expects $P_{\mathrm{t}}(L) \rightarrow 1 / N_{\perp}$, where $N_{\perp}$ is the total number of transverse modes. For finite-length samples $P_{\mathrm{t}}(L)$ is expected to approach this asymptotic value from above, starting from $P_{\mathrm{t}}(0)=1$ in the limit of zero sample length. For the disordered DWN system our data in Fig. 4 (top) indeed indicates a monotonous decrease in $P_{\mathrm{t}}(L)$ with $L$ and a saturation at large $L$ for disorder strengths $K>4$. Although no saturation could be observed for weaker disorder strengths at the system sizes we could access in our numerical calculations, we found no sign that $P_{\mathrm{t}}(L)$ behaves differently for $K<4$, consistent with a flow to a diffusive fixed point even for weak disorder. On the other hand, if weak disorder were an irrelevant perturbation (as it is in the case of a single Weyl node) and the pseudodiffusive fixed point were stable, we would expect that an initial decrease in $P_{\mathrm{t}}(L)$ with $L$ would be compensated by an increase in $P_{\mathrm{t}}(L)$ at larger lengths, a behavior that we confirmed for the weakly disordered SWN (data not shown).

As long as $P_{\mathrm{t}} \gg 1 / N_{\mathrm{t}}$, where $N_{\mathrm{t}} \sim h G / e^{2} \sum_{\mathbf{k}_{\perp}}\left|t\left(\mathbf{k}_{\perp}, 0\right)\right|^{2}$ is the (effective) number of transverse modes participating in the transmission, a condition that is met for the entire parameter range we consider, we expect that $P_{\mathrm{t}}(L)$ has the functional form

$$
P_{\mathrm{t}}(L)=e^{-\left(L-L_{c}\right) / L^{\star}},
$$

where the characteristic length scale $L^{\star}$ can be identified with the mean free path and $L_{c}$ is a length scale that accounts for transient effects at the sample-lead boundary, leading to a quick initial decrease in $P_{\mathrm{t}}$ for short lengths, visible, in particular, for $K \lesssim 4$. The bottom panel in Fig. 4 shows fits of $L^{\star}$ based on the large- $L$ asymptotics of $P_{\mathrm{t}}(L)$. The $K$ dependence of $L^{\star}$ is consistent with the expectation based on Eq. (8), $L^{\star} \sim$ $\hbar v / \Gamma \sim \hbar v / \Lambda e^{A / K}$. We disregard the data points at $K=1$ and $K=2$, for which no reliable asymptotic large- $L$ fit could be made.

The curves for the difference $\Delta R(L)$ in the resistances between the clean and the disordered cases in Fig. 3 can be understood in terms of a crossover from pseudodiffusive to diffusive transport as well. The length scale $L^{\star}(K)$ roughly coincides with the length scale where the second derivative of the resistance-vs-sample length curve vanishes.

For the weakest disorder strength we consider that the maximum sample length $L_{\max }$ is still much smaller than the characteristic length $L^{*}$ of the pseudodiffusive-to-diffusive crossover. For this disorder strength, pseudodiffusive behavior prevails for all system sizes we consider, albeit with a resistance that is slighlty smaller than $R_{0}$. A decrease in the resistivity has also been observed as a finite-size effect for an SWN at weak disorder strengths [13]. A systematic decrease in the resistivity could in principle arise as a consequence of a disorder-induced renormalization of the parameters $v$ and $\eta$ in Hamiltonian (1). For a bulk system, the renormalized parameters $v_{\text {eff }}$ and $\eta_{\text {eff }}$ can be calculated in the Born approximation, which yields an increased effective length scale $\eta_{\text {eff }}>\eta$. Replacing $\eta$ with $\eta_{\text {eff }}$ in the expression for clean conductivity of a finite system, $\sigma_{0, \text { eff }}=e^{2} /\left(2 \pi h \eta_{\text {eff }}\right)$ predicts an increase in the resistance, in conflict with our numerical observation. We conclude that a disorder-induced renormalization of the parameters $v$ and $\eta$ is not the explanation for the observed decrease in resistivity. A more careful analysis of the finite-size effects could be attempted along the lines of that in Ref. [34].

For strong disorder, $K \gtrsim 4$, the characteristic length scale $L^{\star}(K)$ drops below $L_{\max }$ and diffusive behavior can be observed (see, e.g., the resistance data for $L / \eta \gtrsim 40$ and $K=14$ ). Such a diffusive regime is also commonly found in other topological semimetals, such as a two-dimensional Dirac or a three-dimensional simple Weyl node: Although disorder tends to decrease the mean free path, the conductance is still increased by the disorder-induced increase in the density of states, while the band topology and, in three dimensions, standard single-parameter scaling arguments prohibit Anderson localization $[13,31,35]$.

\section{CONCLUSION}

We have investigated the effects of potential disorder for a double Weyl node (DWN), using numerically exact quantum transport simulations in a mesoscopic setup for chemical potential at the nodal point as well as density of states calculations based on the self-consistent Born approximation and the kernel polynomial method (KPM) for a range of energies. Our findings indicate that disorder physics in a DWN is more conventional than in its linearly dispersing counterpart with unit chiral charge, which features a disorder-induced quantum phase transition with the density of states at zero energy as an order parameter. In the DWN, any finite disorder strength induces a finite quasiparticle lifetime $\tau$ at the nodal point. Our numerical and analytical calculations are consistent with previous predictions by Goswami and Nevidomskyy, indicating that the lifetime $\tau$ is exponentially large in the inverse disorder strength [25].

Unfortunately, a quantitative comparison of our calculations for the density of states and our transport simulations is hindered by the fact that only the self-consistent Born approximation (SCBA) can give an estimate of the quasiparticle lifetime $\tau$. However, since the SCBA density of states does not agree quantitatively with the data from the KPM at $\varepsilon=0$, we must also discard its predicted value of $\tau$ for quantitative checks. The density of states, as simulated by the KPM, is, however, a quantity integrated over $k$ space [see Eq. (7)] and cannot be translated into a value of $\tau$ without further assumptions.

In Ref. [33], the disorder-induced phase transition point in a single Weyl node was identified using the condition of scale invariance of the (median) conductance. We repeated a similar analysis with conductance data obtained for the disordered DWN in Sec. III but could not find a scale-invariant point (data not shown). This is consistent with the absence of a disorder-induced phase transition in a DWN band structure. 
For technical convenience, we have used a model for the DWN with continuous rotational symmetry $\left[\eta_{x}=\eta_{y}=\eta\right.$ in Eq. (1)]. In additional numerical calculations we checked that our conclusions do not qualitatively change when $\eta_{x} \neq \eta_{y}$ and the rotational symmetry is reduced to be fourfold.

\section{ACKNOWLEDGMENTS}

We thank J. Dreger for support in the computations done on the HPC cluster of Fachbereich Physik at Freie Universität Berlin. We gratefully acknowledge discussions with J. Reuther and A. Rosch as well as financial support from the Helmholtz Virtual Institute "New States of Matter and Their Excitations" and CRC/Transregio 183 (Project A02) of the Deutsche Forschungsgemeinschaft.

\section{APPENDIX: SCBA EQUATIONS}

Using the identities $\int_{0}^{2 \pi} d \theta \cos (2 \theta) \exp [x \cos [\phi-\theta]]=$ $2 \pi \cos (2 \phi) I_{2}(x)$ and $\int_{0}^{2 \pi} d \theta \exp [x \cos [\phi-\theta]]=2 \pi I_{0}(x)$, where $I_{k}(x)$ is the modified Bessel function of the $k$ th kind, we find the following self-consistency equations from Eq. (5) with ansatz (6),

$$
\begin{aligned}
M\left(P, P_{z}\right)= & \frac{-K r}{(2 \pi)^{2}} \int_{0}^{\infty} d Q \int_{-\infty}^{\infty} d Q_{z}\left[Q^{2} / 2\right. \\
& \left.+M\left(Q, Q_{z}\right)\right] I_{2}\left(Q P r^{2}\right), \\
M_{z}\left(P, P_{z}\right)= & \frac{-K r}{(2 \pi)^{2}} \int_{0}^{\infty} d Q \int_{-\infty}^{\infty} d Q_{z}\left[Q_{z}\right. \\
& \left.+M_{z}\left(Q, Q_{z}\right)\right] I_{0}\left(Q P r^{2}\right), \\
M_{0}\left(P, P_{z}\right)= & \frac{K r}{(2 \pi)^{2}} \int_{0}^{\infty} d Q \int_{-\infty}^{\infty} d Q_{z}\left[M_{0}\left(Q, Q_{z}\right)\right. \\
& -i E] I_{0}\left(Q P r^{2}\right),
\end{aligned}
$$

where

$$
U\left(Q, Q_{z}\right)=Q \frac{\exp \left[-r^{2}\left(P^{2}+Q^{2}+\left(Q_{z}-P_{z}\right)^{2}\right) / 2\right]}{\left[Q^{2} / 2+M\left(Q, Q_{z}\right)^{2}\right]+\left[Q_{z}+M_{z}\left(Q, Q_{z}\right)\right]^{2}-\left[E+i M_{0}\left(Q, Q_{z}\right)\right]^{2}}
$$

and $r=\xi / \eta, E /(\hbar v / \eta)=\varepsilon, M\left(Q=q \eta, Q_{z}=q_{z} \eta\right) \equiv m\left(q, q_{z}\right) \eta$, and analogously for $M_{z}$ and $M_{0}$. Equations (A1) to (A3) can be solved numerically by iteration.

[1] B. A. Bernevig, Nat. Phys. 11, 698 (2015).

[2] S.-Y. Xu, I. Belopolski, N. Alidoust, M. Neupane, G. Bian, C. Zhang, R. Sankar, G. Chang, Z. Yuan, C. Lee, S. Huang, H. Zheng, D. Sanchez, B. Wang, A. Bansil, F. Chou, P. Shibayev, H. Lin, S. Jia, and M. Z. Hasan, Science 349, 613 (2015).

[3] B. Q. Lv, N. Xu, H. M. Weng, J. Z. Ma, P. Richard, X. C. Huang, L. X. Zhao, G. F. Chen, C. Matt, F. Bisti, V. Strokov, J. Mesot, Z. Fang, X. Dai, T. Qian, M. Shi, and H. Ding, Nat. Phys. 11, 724 (2015).

[4] S.-Y. Xu, I. Belopolski, D. Sanchez, C. Guo, G. Chang, C. Zhang, G. Bian, Z. Yuan, H. Lu, Y. Feng, T. Chang, P. Shibayev, M. Prokopovych, N. Alidoust, H. Zheng, C. Lee, S. Huang, R. Sankar, F. Chou, C. Hsu, H. Jeng, A. Bansil, T. Neupert, V. Strocov, H. Lin, S. Jia, and M. Z. Hasan, Sci. Adv. 1, e1501092 (2015).

[5] S. Borisenko, D. Evtushinsky, Q. Gibson, A. Yaresko, T. Kim, M. N. Ali, B. Buechner, M. Hoesch, and R. J. Cava, arXiv:1507.04847v1.

[6] J. Y. Liu, J. Hu, D. Graf, S. M. A. Radmanesh, D. J. Adams, Y. L. Zhu, G. F. Chen, X. Liu, J. Wei, I. Chiorescu, L. Spinu, and Z. Q. Mao, arXiv:1507.07978v1.

[7] C. Zhang, S.-Y. Xu, I. Belopolski, Z. Yuan, Z. Lin, B. Tong, G. Bian, N. Alidoust, C.-C. Lee, S.-M. Huang, T.-R. Chang, G. Chang, C.-H. Hsu, H.-T. Jeng, M. Neupane, D. S. Sanchez, H. Zheng, J. Wang, H. Lin, C. Zhang, H.-Z. Lu, S.-Q. Shen, T. Neupert, M. Z. Hasan, and S. Jia, Nat. Commun. 7, 10735 (2016).

[8] C. Zhang, Z. Lin, C. Guo, S.-Y. Xu, C.-C. Lee, H. Lu, S.-M. Huang, G. Chang, C.-H. Hsu, H. Lin, L. Li, C. Zhang, T. Neupert, M. Z. Hasan, J. Wang, and S. Jia, arXiv:1507.06301v1.
[9] C. Shekhar, A. K. Nayak, Y. Sun, M. Schmidt, M. Nicklas, I. Leermakers, U. Zeitler, Y. Skourski, J. Wosnitza, Z. Liu, Y. Chen, W. Schnelle, H. Borrmann, Y. Grin, C. Felser, and B. Yan, Nat. Phys. 11, 645 (2015).

[10] J. Ruan, S.-K. Jian, H. Yao, H. Zhang, S.-C. Zhang, and D. Xing, Nat. Commun. 7, 11136 (2016).

[11] J. Ruan, S.-K. Jian, D. Zhang, H. Yao, H. Zhang, S.-C. Zhang, and D. Xing, Phys. Rev. Lett. 116, 226801 (2016).

[12] P. Baireuther, J. M. Edge, I. C. Fulga, C. W. J. Beenakker, and J. Tworzydlo, Phys. Rev. B 89, 035410 (2014).

[13] B. Sbierski, G. Pohl, E. J. Bergholtz, and P. W. Brouwer, Phys. Rev. Lett. 113, 026602 (2014).

[14] E. Fradkin, Phys. Rev. B 33, 3263 (1986).

[15] P. Goswami and S. Chakravarty, Phys. Rev. Lett. 107, 196803 (2011).

[16] K. Kobayashi, T. Ohtsuki, K.-I. Imura, and I. F. Herbut, Phys. Rev. Lett. 112, 016402 (2014).

[17] Y. Ominato and M. Koshino, Phys. Rev. B 89, 054202 (2014).

[18] S. V. Syzranov, L. Radzihovsky, and V. Gurarie, Phys. Rev. Lett. 114, 166601 (2015).

[19] S. V. Syzranov, V. Gurarie, and L. Radzihovsky, Phys. Rev. B 91, 035133 (2015).

[20] C. Fang, M. J. Gilbert, X. Dai, and B. A. Bernevig, Phys. Rev. Lett. 108, 266802 (2012).

[21] C. Z. Chen, J. Song, H. Jiang, Q. F. Sun, Z. Wang, and X. C. Xie, Phys. Rev. Lett. 115, 246603 (2015).

[22] G. Xu, H. Weng, Z. Wang, X. Dai, and Z. Fang, Phys. Rev. Lett. 107, 186806 (2011).

[23] S.-M. Huang, S.-Y. Xu, I. Belopolski, C.-C. Lee, G. Chang, B. Wang, N. Alidoust, M. Neupane, H. Zheng, D. Sanchez, 
A. Bansil, G. Bian, H. Lin, and M. Z. Hasan, Proc. Natl. Acad. Sci. USA 113, 1180 (2016).

[24] X. Dai, H.-Z. Lu, S.-Q. Shen, and H. Yao, Phys. Rev. B 93, 161110 (2016).

[25] P. Goswami and A. H. Nevidomskyy, Phys. Rev. B 92, 214504 (2015).

[26] S. Bera, J. D. Sau, and B. Roy, Phys. Rev. B 93, 201302 (2016).

[27] H. Shapourian and T. L. Hughes, Phys. Rev. B 93, 075108 (2016).

[28] A. Weisse, G. Wellein, A. Alvermann, and H. Fehske, Rev. Mod. Phys. 78, 275 (2006).

[29] J. Tworzydlo, B. Trauzettel, M. Titov, A. Rycerz, and C. W. J. Beenakker, Phys. Rev. Lett. 96, 246802 (2006).
[30] Y. Nazarov and Y. Blanter, Theory of Quantum Transport (Cambridge University Press, Cambridge, UK, 2009).

[31] J. H. Bardarson, J. Tworzydło, P. W. Brouwer, and C. W. J. Beenakker, Phys. Rev. Lett. 99, 106801 (2007)

[32] S. Adam, P. W. Brouwer, and S. Das Sarma, Phys. Rev. B 79 , 201404 (2009).

[33] B. Sbierski, E. J. Bergholtz, and P. W. Brouwer, Phys. Rev. B 92, 115145 (2015).

[34] A. Schuessler, P. M. Ostrovsky, I. V. Gornyi, and A. D. Mirlin, Phys. Rev. B 79, 075405 (2009).

[35] K. Nomura, M. Koshino, and S. Ryu, Phys. Rev. Lett. 99, 146806 (2007). 